Short Research Communication

\title{
Reciprocal Interference between Lactobacillus spp. and Gardnerella vaginalis on Initial Adherence to Epithelial Cells
}

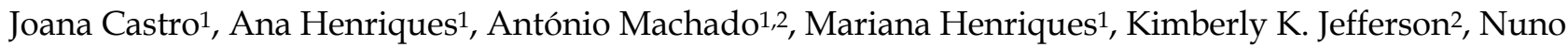
Cerca $^{1 凶}$

1. IBB - Institute for Biotechnology and Bioengineering, Centre of Biological Engineering, University of Minho, Campus de Gualtar 4710-057, Braga, Portugal.

2. Department of Microbiology and Immunology, Virginia Commonwealth University, Richmond, Virginia, USA.

$\triangle$ Corresponding author: nunocerca@ceb.uminho.pt. Tel.: (+351) 253604 400; Fax: (+351) 253604429.

( ) Ivyspring International Publisher. This is an open-access article distributed under the terms of the Creative Commons License (http://creativecommons.org/ licenses/by-nc-nd/3.0/). Reproduction is permitted for personal, noncommercial use, provided that the article is in whole, unmodified, and properly cited.

Received: 2013.03.23; Accepted: 2013.06.17; Published: 2013.07.20

\begin{abstract}
Bacterial vaginosis $(\mathrm{BV})$ is the most common vaginal disorder in women of child-bearing age. It is widely accepted that the microbial switch from normal microflora to the flora commonly associated with $B V$ is characterized by a decrease in vaginal colonization by specific Lactobacillus species together with an increase of $G$. vaginalis and other anaerobes. However, the order of events leading to the development of $\mathrm{BV}$ remains poorly characterized and it is unclear whether the decrease in lactobacilli is a cause or a consequence of the increase in the population density of anaerobes. Our goal was to characterize the interaction between two Gardnerella vaginalis strains, one of which was isolated from a healthy woman (strain 5-I) and the other from a woman diagnosed with BV (strain (0I), and vaginal lactobacilli on the adherence to cervical epithelial cells. In order to simulate the transition from vaginal health to $B V$, the lactobacilli were cultured with the epithelial cells first, and then the $G$. vaginalis strain was introduced. We quantified the inhibition of $G$. vaginalis adherence by the lactobacilli and displacement of adherent lactobacilli by $G$. vaginalis. Our results confirmed that pathogenic $G$ vaginalis I0I had a higher capacity for adhesion to the cervical epithelial cells than strain 5-I. Interestingly, strain I0I displaced L. crispatus but not $L$. iners whereas strain 5-I had less of an effect and did not affect the two species differently. Furthermore, $L$. iners actually enhanced adhesion of strain $10 \mathrm{I}$ but not of strain $5-\mathrm{I}$. These results suggest that BV-causing $G$. vaginalis and $L$. iners do not interfere with one another, which may help to explain previous reports that women who are colonized with $L$. iners are more likely to develop BV.
\end{abstract}

Key words: Lactobacillus spp.; Gardnerella vaginalis; Initial adhesion; Bacterial vaginosis.

\section{Introduction}

It is well established that microbial communities have a strong influence on human health and quality of life. It is not surprising that the bacterial community within the human vagina has a profound impact in women's health, since the microflora present in the vagina play a crucial role in determining the biochemical profile of the vagina and its inflammatory profile [1]. The healthy vagina is predominated by lactobacilli. A number of Lactobacillus species, including L. crispatus, L. gasseri, L. jensenii, L. vaginalis, and L. iners, are common vaginal colonizers [2]. The species differ with respect to their ability to maintain a stable population through environmental changes such as $\mathrm{pH}$ changes due to sexual intercourse or menstruation and with respect to their ability to exclude other bacteria. All of the species produce lactic 
acid and many produce hydrogen peroxide and bacteriocins as well, and these compounds can inhibit the growth of other bacterial species. In addition to inhibiting growth, lactobacilli may be able to interfere with adherence of other species to vaginal epithelial cells. For example, they may produce compounds that inhibits adherence by other species, or may interfere sterically. L. crispatus appears to be one of the most stable and exclusive species, whereas L. iners appears to be less stable and less exclusive. Consequently, women who are colonized by L. crispatus have a decreased risk of developing the vaginal disorder bacterial vaginosis and women who are colonized by $L$. iners have an increased risk [3]. Bacterial vaginosis (BV) is the leading vaginal disorder in women of reproductive age worldwide, contributing for more than $60 \%$ of vulvovaginal infections. It is linked to serious public health consequences including pelvic inflammatory disease, postoperative infections, acquisition and transmission of the HIV virus, and preterm birth [4]. The high prevalence, high relapse rate, and associated complications, make this disorder of paramount global importance [5]. During BV, beneficial lactic acid-producing bacteria (lactobacilli) are replaced by amine-producing anaerobic bacteria. Although BV is considered a polymicrobial condition, one of the predominant bacterial species is often Gardnerella vaginalis [6]. However, the role of this species in the initiation and progression of the disorder is not yet fully understood [1]. Recently it has been described that the vaginal microflora, in cases of $\mathrm{BV}$, forms a multi-species biofilm in which $G$. vaginalis is the dominant bacterial strain [7]. Biofilms are bacterial structures attached to a surface and embedded in a protective matrix, and are known to be more resistant than planktonic cells to the host immune response and also to antibiotic therapy [8]. Of importance, G. vaginalis biofilms were recently characterized by an increased tolerance to hydrogen peroxide and lactic acid when compared to planktonic cells [9]. However, it is not clear whether some event causes a drop in the population of lactobacilli, which then makes conditions permissive to the growth of other bacteria, whether BV-associated species such as G. vaginalis displace the lactobacilli, or whether these two factors are not directly related [10]. Understanding the interactions between commensal lactobacilli and the anaerobes that characterize the vaginal ecosystem in cases of BV is of extreme importance to help unravel the aetiology, pathogenesis, and progression of this condition. Adhesion to host cells is a critical initial step in any infectious process. Interference studies, using in vitro models of infection have been used extensively for the study of the interactions between commensal bacteria and pathogenic bacteria [11-13], and, more specifically to study the ability of lactobacilli to block adhesion of pathogenic bacteria to the vaginal epithelium [14-17]. The main goal of our work was to evaluate the reciprocal effects between $G$. vaginalis and $L$. crispatus or L. iners during initial adhesion to cervical epithelial cells.

\section{Materials and Methods}

\section{Strains and culture conditions}

G. vaginalis strain 5-1 was isolated from a woman without BV and strain 101 from a woman with BV, diagnosed by the Nugent Gram stain scoring system [18]. Both G. vaginalis strains were collected from swabs specimens at Brigham and Women's Hospital, Boston MA, USA [19] The Lactobacillus spp. used were L. crispatus EX533959VC06, and L. iners ATCC 55195. L. iners and both G. vaginalis were grown in supplement Brain Heart Infusion (BHI) (Oxoid) containing $2 \%(\mathrm{w} / \mathrm{w})$ gelatin (Oxoid), $0,5 \%$ yeast extract (Liofilchem), 0,1\% starch (FisherScientific), and 0,1\% glucose (Liofilchem)). L. crispatus was grown in Man Rogosa and Sharpe both (MRS) (Sigma). All cultures were grown at $37^{\circ} \mathrm{C}$ for $48 \mathrm{~h}$ under anaerobic conditions, using the Anaerogen pack system (Oxoid).

Bacterial suspensions were collected by centrifugation at 7,197 $\times \mathrm{g}$ at $4^{\circ} \mathrm{C}$ for $10 \mathrm{~min}$. The culture medium was discarded, and the bacteria were washed once with phosphate buffered saline (PBS). Bacteria were resuspended in Dulbecco's modified Eagle's medium (DMEM) medium (Sigma-Aldrich) and the optical density at $600 \mathrm{~nm}\left(\mathrm{OD}_{600}\right)$ was determined. Correlations between $\mathrm{OD}_{600}$ and Colony Forming Units (CFU) were made prior to the experiments, and the DMEM suspensions were adjusted to concentrations of either $1 \times 10^{3}$ or $1 \times 10^{9} \mathrm{CFU} / \mathrm{mL}$ before use in the adhesion assays.

\section{Culture of HeLa cell line}

HeLa epithelial cells (American Tissue Culture Collection, ATCC CCL-2) were cultured in DMEM supplemented with 15\% (vol/vol) FBS (Sigma) and 1 $\mathrm{IU}$ penicillin/streptomycin $\mathrm{mL}^{-1}$ (Sigma) at $37^{\circ} \mathrm{C}$ and in $5 \% \mathrm{CO}_{2}$ (vol/vol). Cells were cultured in 24-well tissue culture plates (Orange Scientific) containing 12 $\mathrm{mm}$ glass slides (Marienfeld) until they reached a density of $2 \times 10^{5}$ cells per well $(\approx 90 \%$ confluence), at $37^{\circ} \mathrm{C}$ and $5 \% \mathrm{CO}_{2}$. Before the competition and interference of adhesion assays, the cells were washed twice with $500 \mu$ l of sterile PBS (Sigma) to remove non adherent cells and culture media.

\section{Adhesion assays}

Two distinct experiments were performed to study the interactions between lactobacilli and $G$. 
vaginalis during adhesion to epithelial cells: competition and interference assays.

For the competition assays, equal volumes of the optimized concentrations of each of the Lactobacillus strains or each of the $G$. vaginalis strains were added simultaneously to each well of the 24 -well plate containing the HeLa monolayers. The plates were incubated for $30 \mathrm{~min}$ at $37^{\circ} \mathrm{C}$ in anaerobic conditions, at 0,081 $g$ (PSU-10i, Biosan).

For the interference assays two distinct cell densities of each Lactobacillus species were added to each well of the 24-well containing the monolayer. The plates were incubated for $4 \mathrm{~h}$ at $37{ }^{\circ} \mathrm{C}$ in anaerobic conditions and $0,081 \mathrm{~g}$. Non adherent lactobacilli were removed by washing with $500 \mu \mathrm{l}$ of sterile PBS and subsequently $G$. vaginalis was incubated with the monolayer for $30 \mathrm{~min}$ at $37^{\circ} \mathrm{C}$ in anaerobic conditions and $0,081 \mathrm{~g}$. In both assays each well was carefully washed twice with $500 \mu \mathrm{l}$ of sterile PBS to remove non-adherent bacteria. In each assay, adhesion controls for each bacteria alone were performed simultaneously using the same experimental conditions. Adherence assays were repeated three independent times, with technical triplicate samples each time.

Bacterial quantification was done as we previously described [20]. Briefly, after fixing with methanol, a Peptide Nucleic Acid (PNA) probe, that we previously developed, specific for G. vaginalis, was used [20]. An additional staining step was done at the end of the hybridization procedure, covering each glass slide with $20 \mu \mathrm{L}$ of DAPI $(2.5 \mu \mathrm{g} / \mathrm{mL})$ (Sigma). Then each slide was immediately observed using fluorescence microscopy. All these assays were repeated three independent times, with technical triplicate samples each time. In each experimental assay, a negative control was performed simultaneously in which all the steps described above were carried out, but where no probe or DAPI staining were added in the hybridization step. Microscopic visualization was performed using an Olympus BX51 epifluorescence microscope equipped with a CCD camera (DP72; Olympus) and filters capable of detecting the PNA probe (BP 530-550, FT 570, LP 591 sensitive to the Alexa Fluor 594 molecule attached to the Gard162 probe). Also, DAPI staining was detected by an appropriate filter, more exactly, BP 365-370, FT 400, LP 421 present in the microscope. All adhesion assays were quantified using DAPI for total cell count and Gard162 probe for G. vaginalis quantification. Results were expressed as the average number of $G$. vaginalis and Lactobacillus spp. per epithelial cell, defined as the adhesion index. The number of $G$. vaginalis was compared with respective control values (i.e. microbial cells without lactobacilli) and the number of Lactobacillus spp. was also compared with control values (microbial cells without G. vaginalis). The control values were taken as $100 \%$ of adhesion and the percentage of adhesion of $G$. vaginalis and Lactobacillus spp. was calculated in comparison with respective controls.

\section{Statistical Analysis}

The data were analyzed using a two-tailed ANOVA or Student's t-test with SPSS statistical software (version 17.0) and expressed as mean \pm standard deviation (SD). $\mathrm{P}<0.05$ was considered significant.

\section{Results and discussion}

\section{Initial adhesion competition assays to HeLa cells}

In the present study we used two previously characterized G. vaginalis strains, a non-BV isolate, 5-1 and a BV isolate, 101 [21], and evaluated the interactions with $L$. crispatus and L. iners. Since quantitative initial adhesion studies often require optimization steps in order to obtain a robust in vitro system [22] the optimal initial inocula and time of adhesion for this study were first tested using initial inocula ranged from $1 \mathrm{E} 3$ to $1 \mathrm{E} 9 \mathrm{CFU} / \mathrm{mL}$ and the time of adhesion ranged from 10 to 240 minutes. The absolute adhesion levels of bacteria starting from inocula of 1E5 $\mathrm{CFU} / \mathrm{ml}$ were not significantly different than those of inocula at 1E3 CFU/mL (data not shown). The same occurred between 1E8 and 1E9 CFU/mL (data not shown). In the follow up studies, only the higher and/or lower inocula concentrations were used.

To determine bacterial fitness in the initial adhesion to the HeLa epithelial cells, combinations of each G. vaginalis and Lactobacillus strains were used in initial adhesion competition assays. Suspensions with 1E9 $\mathrm{CFU} / \mathrm{mL}$ of both lactobacilli and $\mathrm{G}$. vaginalis strains were incubated together $\left(37^{\circ} \mathrm{C}, 30 \mathrm{~min}\right)$ in order to evaluate the interference of bacterial adhesion to a monolayer of epithelial cells, and were compared to the initial adhesion of the individual bacterial species. Both lactobacilli and G. vaginalis strains used in the present study were able to adhere, to different degrees, to epithelial cells (Figure 1). G. vaginalis 101 exhibited the highest adhesion potential. Interestingly, L. crispatus and L. iners both significantly reduced adherence of the $G$. vaginalis strains and to similar extents. These results are in agreement with Boris et al [23] and Atassi et al [22], demonstrating that different Lactobacillus strains from vaginal microbiota are able to interfere with $G$. vaginalis strains when in competition for adhesion to epithelial HeLa cells. On the other hand, interference with Lactobacillus adherence was notably different depending on the G. vaginalis strain 
used: interference caused by G. vaginalis 5-1 was relatively mild and the interference with both species of lactobacilli was similar. Conversely, strain 101 reduced L. crispatus dramatically but did not interfere substantially with $L$. iners. A limitation of our study was the use of just one strain of each L. crispatus and $L$. iners, and we cannot account for strain to strain variability. Also, we only used two representative Lactobacillus species, one that correlates with health, and one that is associated with $\mathrm{BV}$, and we did not explore how other lactobacilli species would interact with both G. vaginalis [24].

\section{Initial adhesion interference assays to HeLa cells}

At least three plausible scenarios for the progression of BV exist. Either some event leads to a decline in lactobacilli colonization levels and this allows overgrowth of other species, overgrowth on the $\mathrm{BV}$-associated species leads to the decrease of the beneficial lactobacilli, or both processes occur simultaneously $[18,25]$. In the healthy urogenital tract of adult females, it is supposed that the indigenous lactobacilli block the colonization of pathogenic bacteria by occupying or masking (by steric hindrance) their potential binding sites in the mucosa [26]. To simulate a decline in lactobacilli, we allowed both low and high numbers of lactobacilli to pre-adhere to epithelial monolayers and then determined their effect on adherence of $G$. vaginalis. To determine whether $G$. vaginalis is able to displace pre-established lactobacilli populations, we also quantified the effect of $G$. vaginalis on adherence of the lactobacilli. At the high dose, an average of $13.65 \mathrm{~L}$. crispatus and 13.89 L. iners adhered per HeLa cell and at low dose and average of 1.86 L. crispatus and 0.95 L. iners adhered per HeLa cell (Table 1). G. vaginalis strains were subsequently added and incubation was continued for further $30 \mathrm{~min}$.

B

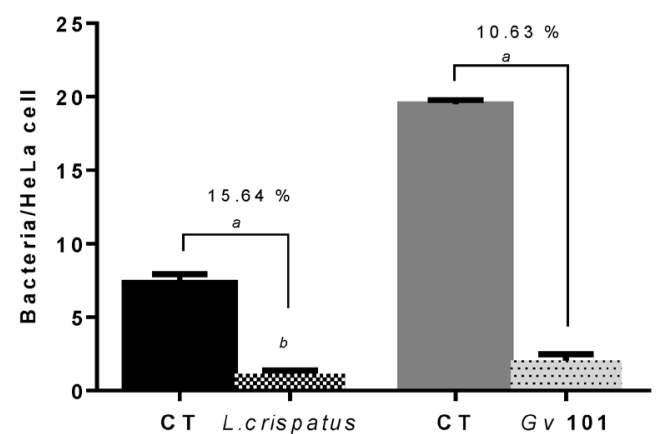

D

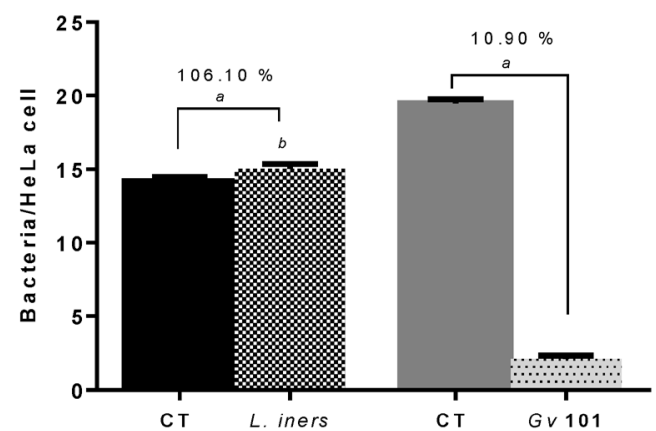

Figure I. Competition between Lactobacillus spp. and G. vaginalis initial adhesion to HeLa epithelial cells. The percentage of adhesion is the result of the variation in the adhesion of Lactobacillus spp. and G. vaginalis strains to epithelial cells in comparison to controls (control values, $100 \%$ of adhesion) when incubated alone at the same conditions. Control (CT) value of respective Lactobacillus sp.; , Control (CT) value of respective $G$. vaginalis strain. (A) $\otimes$. High levels of $L$. crispatus and ...., high levels of $G$. vaginalis $5-I$. (B) $\approx$ High levels of $L$. crispatus and ..., high levels of $G$. vaginalis $I 0 I$.

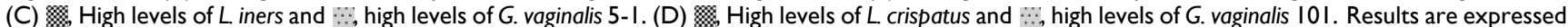
as bacteria/HeLa cell and the data presented are the mean \pm SD of three independent assays. ${ }^{a}$ Values significantly different from the respective control, $\mathrm{p}<0.05$ when using T-student statistical analysis ( $95 \%$ confidence interval) for comparison of control and test of $G$. vaginalis adhesion. ${ }^{b} p<0.05$ analysed using ANOVA Tukey statistical test ( $95 \%$ confidence interval) for comparison of adhesion between assays from G. vaginalis $5-\mathrm{I}$ and I0I at same conditions. 
Table I. Influence of $L$. crispatus and $L$. iners on G. vaginalis initial adhesion into HeLa cells. Two inocula of $I \times 10^{9}$ and $I \times 10^{3}$ $\mathrm{CFU} / \mathrm{mL}$ from each Lactobacillus sp. were pre-adhered to the epithelial cells, averaging I3.65 L. crispatus and I3.89 L. iners or I.86 L. crispatus and $0.95 \mathrm{~L}$. iners adhered per HeLa cell, respectively. The two $G$. vaginalis strains were added subsequently. In addition, $G$. vaginalis control was elaborated in each assay without Lactobacillus sp. pre-adhesion step, averaging $0.94 \mathrm{G}$. vaginalis $5-\mathrm{I}$ and I.06 G. vaginalis I0I adhered per HeLa cell. In the "Influence of vaginal adhering Lactobacillus sp. strains on adhesion of $G$. vaginalis" column, the percentage of adhesion to cells colonized by the lactobacilli relative to HeLa cells alone is shown. In the "Displacement of Lactobacillus sp. pre-adhered to HeLa monolayer" column, the percentage of lactobacilli that remained adherent following $G$. vaginalis challenge relative to unchallenged epithelial cells is shown. Results were expressed as the average number of $G$. vaginalis and Lactobacillus sp. per epithelial cell, defined as the adhesion index.

\begin{tabular}{|c|c|c|c|c|c|c|c|c|c|}
\hline \multirow{3}{*}{ G. vaginalis } & & \multicolumn{4}{|c|}{$\begin{array}{l}\text { Influence of vaginal adhering Lactobacillus sp. } \\
\text { strains on adhesion of G. vaginalis }\end{array}$} & \multicolumn{4}{|c|}{$\begin{array}{l}\text { Displacement of Lactobacillus sp. pre-adhered to } \\
\text { HeLa monolayer }\end{array}$} \\
\hline & & \multicolumn{2}{|c|}{ L. crispatus } & \multicolumn{2}{|c|}{ L. iners } & \multicolumn{2}{|c|}{ L. crispatus } & \multicolumn{2}{|c|}{ L. iners } \\
\hline & & 13.65/HeLa & 1.86/HeLa & 13.89/HeLa & $0.95 / \mathrm{HeLa}$ & 13.65/HeLa & 1.86/HeLa & 13.89/HeLa & 0.95/HeLa \\
\hline $5-1$ & $\begin{array}{l}\text { Adhesion } \\
\text { index }\end{array}$ & $0.14( \pm 0.02)$ & $0.07( \pm 0.07)$ & $0.27( \pm 0.05)$ & $0.80( \pm 0.09)$ & $1.64( \pm 0.54)$ & $0.72( \pm 0.06)$ & $12.13( \pm 0.51)$ & $0.87( \pm 0.04)$ \\
\hline 0.94/HeLa & $\begin{array}{l}\% \text { of } \\
\text { adhesion }\end{array}$ & $15.00 \%{ }^{a}$ & $7.00 \%{ }^{a}$ & $28.60 \%^{a}$ & $84.58 \%$ & $12.00 \%{ }^{a}$ & $39.00 \% a$ & $88.60 \%^{b}$ & $91.45 \%$ \\
\hline 101 & $\begin{array}{l}\text { Adhesion } \\
\text { index }\end{array}$ & $0.05( \pm 0.03)$ & $0.18( \pm 0.19)$ & $1.74( \pm 0.28)$ & $1.5( \pm 0.16)$ & $0.29( \pm 0.28)$ & $0.81( \pm 0.18)$ & $2.63( \pm 0.36)$ & $0.65( \pm 0.00)$ \\
\hline 1.06/HeLa & $\begin{array}{l}\% \text { of } \\
\text { adhesion }\end{array}$ & $7.30 \%{ }^{a}$ & $7.60 \%^{a}$ & $163.30 \%$ & $135.41 \%$ & $19.50 \%{ }^{a}$ & $43.60 \%{ }^{a}$ & $18.98 \% a, b$ & $69.35 \%$ \\
\hline
\end{tabular}

${ }^{a} \mathrm{p}<0.05$ when using T-student statistical analysis ( $95 \%$ confidence interval) for comparison of control and test of $G$. vaginalis adhesion. ${ }^{b} \mathrm{p}<0.05$ analysed using ANOVA Tukey statistical test (95\% confidence interval) for comparison of adhesion between assays from G. vaginalis 5-1 and 101 at same conditions.

As can be seen in Table 1, L. crispatus drastically reduced the adhesion levels of both $G$. vaginalis $5-1$ and 101, irrespective of the number of lactobacilli adhering to the HeLa cells. In addition, both strains of G. vaginalis displaced L. crispatus equally. Interestingly, the same was not true for the interactions with L. iners. A higher dose of L. iners was required to significantly reduce $G$. vaginalis $5-1$ adherence, but $L$. iners actually enhanced pathogenic G. vaginalis 101 adhesion, at both doses. L. iners also resisted the displacement induced by both G. vaginalis in higher extent that $L$. crispatus, suggesting that L. iners is more adaptable to cohabitate with BV associated G. vaginalis strains. Previously, the detection of L. iners in women with and recovering from $B V$ has led to the suggestion that this lactobacilli species was not protective against disease [27-29]. Our results suggest that L. iners can enhance specific strains of G. vaginalis and could be potentially capable to facilitate the development of BV.

\section{Conclusion}

Vaginal lactobacilli play a key role in the inhibition of growth of other bacterial species through the production of lactic acid, hydrogen peroxide, and bacteriocins. However, less is known about the ability of lactobacilli to inhibit adherence of other species. As far as we are aware, this is the first study to quantify the mutual effects of commensal and pathogenic $G$. vaginalis and vaginal lactobacilli on one another with respect to initial adherence to epithelial cells. Adherence is an early step in colonization and the first step of biofilm formation, and therefore plays a criti- cal role in pathogenesis. Lactobacilli could inhibit adherence of BV-causing G. vaginalis through steric hindrance or by masking receptors. However, our finding that an average of fewer than 2 L. crispatus cells per epithelial cells can significantly interfere with G. vaginalis adherence suggests that the size of each bacterial cell may be one of the factors affecting adherence (as L. crispatus cells are larger than L. iners). Understanding the interactions between lactobacilli, that normally compose the healthy microflora, and the anaerobes that characterize the vaginal ecosystem, in cases of $\mathrm{BV}$, is of extreme importance to help unravel the aetiology of this condition [16]. Our study showed a link between lactobacilli and G. vaginalis commonly found in healthy women or in women with BV: while G. vaginalis isolated from a BV patient showed a higher virulence potential, $L$. iners was more resistant to the interference of $G$. vaginalis. Our results can help to explain why L. iners is normally found at higher concentrations in BV patients and provides further evidence for the existence of virulent and non-virulent $G$. vaginalis strains.

\section{Acknowledgements}

This work was supported by European Union funds (FEDER/COMPETE) and by national funds $(\mathrm{FCT})$ under the project with reference FCOMP-01-0124-FEDER-008991 (PTDC/BIA-MIC/ 098228/2008) and in part by funds from the National Institutes of Health (P60-MD002256). AM acknowledges the FCT individual fellowship SFRH/BD/ $62375 / 2009)$. 


\section{Competing Interests}

The authors have declared that no competing interest exists.

\section{References}

1. Brotman RM. Vaginal microbiome and sexually transmitted infections: an epidemiologic perspective. J Clin.Invest 2011; 121:4610-4617

2. Tamrakar R, Yamada T, Furuta I, Cho K, Morikawa M, Yamada H, Sakuragi N, Minakami H. Association between Lactobacillus species and bacterial vaginosis-related bacteria, and bacterial vaginosis scores in pregnant Japanese women. BMC Infect Dis 2007; 7:128

3. Verstraelen $H$, Verhelst $R$, Claeys $G$, De BE, Temmerman $M$, Vaneechoutte $M$. Longitudinal analysis of the vaginal microflora in pregnancy suggests that $L$. crispatus promotes the stability of the normal vaginal microflora and that L. gasseri and/or L. iners are more conducive to the occurrence of abnormal vaginal microflora. BMC.Microbiol. 2009; 9:116

4. Falagas ME, Betsi GI, Athanasiou S. Probiotics for the treatment of women with bacterial vaginosis. Clin.Microbiol.Infect. 2007; 13:657-664

5. Turovskiy Y, Sutyak NK, Chikindas ML. The aetiology of bacterial vaginosis. J Appl.Microbiol 2011; 110:1105-1128

6. Gutman RE, Peipert JF, Weitzen S, Blume J. Evaluation of clinical methods for diagnosing bacterial vaginosis. Obstet.Gynecol. 2005; 105:551-556

7. Swidsinski A, Mendling W, Loening-Baucke V, Ladhoff A, Swidsinski S, Hale LP, Lochs $H$. Adherent biofilms in bacterial vaginosis. Obstet.Gynecol. 2005; 106:1013-1023

8. Hall-Stoodley L, Stoodley P, Kathju S, Hoiby N, Moser C, Costerton JW, Moter A, Bjarnsholt T. Towards diagnostic guidelines for biofilm-associated infections. FEMS Immunol Med Microbiol 2012; 65:127-145

9. Patterson JL, Girerd PH, Karjane NW, Jefferson KK. Effect of biofilm phenotype on resistance of Gardnerella vaginalis to hydrogen peroxide and lactic acid. Am.J.Obstet.Gynecol. 2007; 197:170-177

10. Leppaluoto PA. Bacterial vaginosis: what is physiological in vaginal bacteriology? An update and opinion. Acta Obstet Gynecol Scand. 2011; 90:1302-1306

11. Campana R, Federici S, Ciandrini E, Baffone W. Antagonistic activity of Lactobacillus acidophilus ATCC 4356 on the growth and adhesion/invasion characteristics of human Campylobacter jejuni. Curr.Microbiol. 2012; 64:371-378

12. Mappley LJ, Tchorzewska MA, Cooley WA, Woodward MJ, La Ragione RM. Lactobacilli antagonize the growth, motility, and adherence of Brachyspira pilosicoli: a potential intervention against avian intestinal spirochetosis. Appl.Environ.Microbiol 2011; 77:5402-5411

13. McLean NW, Rosenstein IJ. Characterisation and selection of a Lactobacillus species to re-colonise the vagina of women with recurrent bacterial vaginosis. J Med Microbiol 2000; 49:543-552

14. Farnworth ER. The evidence to support health claims for probiotics. J Nutr. 2008; 138:1250S-1254S

15. Kandler O, Weiss N. Genus Lactobacillus. In: Sneath PH, Mair SN, Sharpe NE, Holt JH eds. Bergey's Manual of Systematic Bacteriology. Baltimore, New York: Williams and Wilkins. 1986: 1209-1234

16. Rosenstein IJ, Fontaine EA, Morgan DJ, Sheehan M, Lamont RF, Taylor-Robinson D. Relationship between hydrogen peroxide-producing strains of lactobacilli and vaginosis-associated bacterial species in pregnant women. Eur.J Clin.Microbiol Infect Dis. 1997; 16:517-522

17. Saxelin M, Tynkkynen S, Mattila-Sandholm T, de Vos WM. Probiotic and other functional microbes: from markets to mechanisms. Curr.Opin.Biotechnol. 2005; 16:204-211

18. Srinivasan S, Fredricks DN. The human vaginal bacterial biota and bacterial vaginosis. Interdiscip.Perspect.Infect.Dis. 2008; 2008:750479

19. Patterson JL, Stull-Lane A, Girerd PH, Jefferson KK. Analysis of adherence, biofilm formation and cytotoxicity suggest a greater virulence potential of Gardnerella vaginalis relaive to other bacterial-vaginosis-associated anaerobes. Microbiology 2010; 156:392-399

20. Machado A, Almeida C, Salgueiro D, Henriques A, Vaneechoutte M, Haesebrouck F, Vieira MJ, Rodrigues L, Azevedo NF, Cerca N. Fluorescence In Situ Hybridization Method Using Peptide Nucleic Acid Probes for rapid detection of Lactobacillus and Gardnerella spp. BMC Microbiology 2013;: 13

21. Harwich MD, Alves JM, Buck GA, Strauss JF, Patterson JL, Oki AT, Girerd PH, Jefferson KK. Drawing the line between commensal and pathogenic Gardnerella vaginalis through genome analysis and virulence studies. BMC Genomics 2010; :11
22. Atassi F, Brassart D, Grob P, Graf F, Servin AL. Lactobacillus strains isolated from the vaginal microbiota of healthy women inhibit Prevotella bivia and Gardnerella vaginalis in coculture and cell culture. FEMS Immunol.Med.Microbiol. 2006; 48:424-432

23. Boris S, Suarez JE, Vazquez F, Barbes C. Adherence of human vaginal lactobacilli to vaginal epithelial cells and interaction with uropathogens. Infect.Immun. 1998; 66:1985-1989

24. Verhelst R, Verstraelen H, Claeys G, Verschraegen G, Van Simaey L, De Ganck C, De Backer E, Temmerman M, Vaneechoutte M. Comparison between Gram stain and culture for the characterization of vaginal microflora: definition of a distinct grade that resembles grade I microflora and revised categorization of grade I microflora. BMC Microbiology 2005; :5

25. Sobel JD. Bacterial vaginosis. Annu.Rev.Med. 2000; 51:349-356

26. Zarate G, Nader-Macias ME. Influence of probiotic vaginal lactobacilli on in vitro adhesion of urogenital pathogens to vaginal epithelial cells. Lett.Appl.Microbiol. 2006; 43:174-180

27. Lopes dos Santos Santiago G, Tency I, Verstraelen H, Verhelst R, Troq M, Temmerman M, Vancoillie L, Decat E, Cools P, Vaneechoutte M. Longitudinal qPCR Study of the Dynamics of L. crispatus, L. iners, A. vaginae, (Sialidase Positive) G. vaginalis, and P. bivia in the Vagina. PLoS One 2012; doi:10.1371/journal.pone.0045281

28. Macklaim JM, Gloor GB, Anukam KC, Cribby S, Reid G. At the crossroads of vaginal health and disease, the genome sequence of Lactobacillus iners AB-1. Proc.Natl.Acad.Sci.U.S.A 2011; 108 Suppl 1:4688-4695

29. Rampersaud R, Planet PJ, Randis TM, Kulkarni R, Aguilar JL, Lehrer JI, Ratner AJ. Inerolysin, a cholesterol-dependent cytolysin produced by Lactobacillus iners. J Bacteriol. 2011; 193:1034-1041. 